\title{
Sirtuin mediated neuroprotection and its association with autophagy and apoptosis: Studies employing transgenic C. elegans model
}

\author{
Aamir Nazir ${ }^{*}$, Pooja Jadiya \\ From Molecular Neurodegeneration: Basic biology and disease pathways \\ Cannes, France. 10-12 September 2013
}

\section{Background}

Sirtuins, NAD+-dependent protein deacetylases, are well known for their role in longevity. Aging is the major known risk factor for the onset of Parkinson's disease (PD). In our previous studies, SIRT1 induction by calorie restriction $(\mathrm{CR})$ diet regimen has been shown to protect against dopaminergic neurodegeneration via sir-2.1 mediated pathway. Taking the studies forward, we endeavoured to understand the role of nicotinamide adenine dinucleotide activated protein deacetylase Sir2p/Sirt1 in calorie restriction mediated prevention of Parkinsonism employing transgenic Caenorhabditis elegans expressing human alpha synuclein.

\section{Materials and methods}

We took advantage of the powerful genetics of model system C. elegans towards achieving our objectives. Transgenic C. elegans models - NL5901 ( $\mathrm{P}_{\text {unc-54::alphasynuclein:: }}$ YFP+unc-119) expressing 'human' alpha synuclein; BZ555 $\left(\mathrm{P}_{\text {dat }-1: \mathrm{GFP})}\right.$ expressing green fluorescence protein (GFP) specifically in eight dopaminergic (DA) neurons and DA2123 expressing LGG-1::GFP, were employed for the studies. Gene silencing was achieved by employing RNAi methodology and the role of sirtuin was assayed by studying various parameters including alpha synuclein aggregation, content of reactive oxygen species, content of lipds, content of mitochondria and content of neurotransmitter dopamine.

\section{Results}

Our findings provide evidences towards the role of calorie restriction in reducing $\alpha$-synuclein aggregation, mitochondrial content, lipid content and ROS in human

CSIR-Central Drug Research Institute, Lucknow, India $\alpha$-synuclein expressing strain of C. elegans. RNAi of sir-2.1 enhanced aggregation of alpha synuclein but sir-2.1 silenced worms raised on reduced calorie diet didn't show protective effect in reducing protein aggregation which proved that protective effects of calorie restriction were mediated by NAD-dependent histone deacetylase activity of $\operatorname{sir}-2.1$. We next focused on the mechanism of the sir-2.1 mediated neuroprotective effect via autophagy. Assessment of autophagy in C. elegans was performed using a transgenic strain DA2123, expressing LGG-1::GFP. Worms with RNAi induced gene silencing of sir-2.1 showed decreased expression of LGG-1::GFP and decreased mRNA level of different autophagy genes including bec-1,atg-5, atg-7, $\operatorname{lgg}-1$ and $\operatorname{atg}-13$ in qPCR studies. In order to further examine the signaling pathways regulating the SIRT1 mediated regulation of autophagic degradation, we analyzed the expression of different apoptosis genes including ced-4, cep-1, lin-35, jkk-1 and jnk-1. In our studies, silencing of sir-2.1 showed significant up-regulation of ced-4 (Apaf-1) and cep-1 (p53 ortholog- DNA damage pathway) apoptosis genes.

\section{Conclusions}

Our study provides evidence for the protective role of sir2.1 on autophagosome formation in C. elegans, which is associated with the p53 and apaf-1 dependent signaling pathways; the well-known stress resistance mediators.

Published: 4 October 2013

doi:10.1186/1750-1326-8-S1-P65

Cite this article as: Nazir and Jadiya: Sirtuin mediated neuroprotection and its association with autophagy and apoptosis: Studies employing transgenic C. elegans model. Molecular Neurodegeneration 2013 8(Suppl 1): P65. 